\title{
ЗАМЕЧАНИЕ О НАИЛУЧШИХ КВАДРАТУРНЫХ ФОРМУЛАХ С ВЕСОВОЙ ФУНКЦИЕЙ
}

M. LEVIN, V. ARRO. MARKUS PARIMATE KAALUFUNKTSIOONIGA KVADRATUURVALEMITE KOHTA

M. LEVIN, V. ARRO. A REMARK ON THE OPTIMAL QUADRATURE FORMULAS WITH WEIGHT FUNCTION

Для приближенного вычисления определенного интеграла от функции $f_{0}(x)$ возможен следующий путь $\left[{ }^{1-2}\right]$. Среди множества квадратурных формул определенного вида выбираем ту, которая в некотором смысле является наилучшей на множестве $H$ функций $f(x)$, содержащем заданную функцию $f_{0}(x)$. Интеграл от $f_{0}(x)$ считаем по выбранной наилучшей формуле.

Пусть $r, n, M, 1 \leqslant q \leqslant \infty$ заданы, $W^{r} L_{q}-$ множество всех функций $f(x)$, которые на отрезке $[0,1]$ имеют абсолютно непрерывную производную порядка $r-1$ и удовлетворяют условию

$$
\left\|f^{(r)}(x)\right\|_{L_{q}(0,1)} \leqslant M .
$$

Для заданной функции $\alpha(x)$ через $W^{r} \alpha(x) L_{q}$ обозначим множество всех непрерывных на $[0,1]$ функций $f(x)$ таких, что $f(\alpha(x)) \in W^{r} L_{q}$.

Через $x_{k}^{*}, A_{k}^{*} \quad(k=1, \ldots, n)$ обозначим узлы и веса наилучшей $\left[{ }^{2}\right]$ на множестве $W^{r} L_{q}$ формулы вида

$$
\int_{0}^{1} f(x) d x=\sum_{k=1}^{n} A_{k} f\left(x_{k}\right)+R_{n}(f),
$$

где $0 \leqslant x_{1}<x_{2}<\ldots<x_{n} \leqslant 1$. Другими словами, будем считать числа $x_{k}=x_{k}^{*}, A_{k}=A_{k}^{*} \quad(k=1, \ldots, n)$ выбранными из условия, чтобы

$$
\sup _{f \in W^{r} L_{q}}\left|R_{n}(f)\right|
$$

имело наименьшее значение.

Существенно отметить, что числа $\left\{x_{k}^{*}, A_{k}^{*}\right\}$ не зависят от величины $M$ и для ряда значений $r$ известны $\left[{ }^{2}\right]$.

Будем считать, что заданная функция $\varrho(x)$ суммируема на $[0,1]$ и такова, что функция

$$
\varphi(x)=\frac{1}{v} \int_{0}^{x} \varrho(t) d t,
$$


где $v=\int_{0}^{1} \varrho(t) d t$, монотонно возрастает на $[0,1]$. Через $\lambda(x)$ обозначим функцию, обратную на $[0,1]$ к функции $\varphi(x)$. Например, если $\varrho(x)=$ $=x^{-1 / 2}$, то $\lambda(x)=x^{2}$, а для $\varrho(x)=[x(1-x)]^{-1 / 2}$ легко подсчитать, что $\lambda(x)=\sin ^{2}\left(\frac{\pi}{2} x\right)$.

Теорема. Наилучшая на $\mathbb{W}_{\lambda(x)}^{r} L_{q}$ форлула вида

$$
\int_{0}^{1} \mathrm{Q}(x) f(x) d x=\sum_{k=1}^{n} B_{k} f\left(t_{k}\right)+E_{n}(f) \quad\left(0 \leqslant t_{1}<t_{2}<\ldots<t_{n} \leqslant 1\right),
$$

т. е. формула (2) с наименьшим значением величины

$$
E_{n}=\sup _{f \in W_{\lambda(x)}^{r} L_{q}}\left|E_{n}(f)\right|,
$$

имеет узлы, веса

$$
t_{k}=\lambda\left(x_{k}^{*}\right), \quad B_{k}=v A_{k}^{*} \quad(k=1,2, \ldots, n)
$$

и оценку остатка

$$
E_{n}=v \cdot \inf _{\left\{x_{k}, A_{k}\right\}} \sup _{f \in W^{r} L_{q}}\left|R_{n}(f)\right| .
$$

Д ок аз а тельство. Пусть $f(x)$ непрерывна на $[0,1], \quad F_{f}(x)=$ $=f(\lambda(x))$. Тогда, учитывая обозначения (в том числе и $(1))$, имеем

$$
\int_{0}^{1} \varrho(x) f(x) d x=v \int_{0}^{1} F_{f}(x) d x=v \sum_{k=1}^{n} A_{k} F_{f}\left(x_{k}\right)+v R_{n}\left(F_{f}\right) .
$$

Очевидно, здесь

$$
\sup _{f \in W_{\lambda(x)^{L} q}^{r}}\left|R_{n}\left(F_{f}\right)\right| \leqslant \sup _{f \in W^{r} L_{q}}\left|R_{n}(f)\right| .
$$

С другой стороны, пусть $\mu(x)-$ произвольная функция из $W^{r} L_{q}$, a $\gamma(x)=\mu(\varphi(x))$. Тогда $\gamma(x) \in W_{\lambda(x)}^{r} L_{q}$.

Кроме того,

$$
\begin{gathered}
R_{n}\left(F_{\gamma}\right)=\int_{0}^{1} \gamma(\lambda(x)) d x-\sum_{k=1}^{n} A_{k} \gamma\left(\lambda\left(x_{k}\right)\right)=\int_{0}^{1} \mu(x) d x- \\
-\sum_{k=1}^{n} A_{k} \mu\left(x_{k}\right)=R_{n}(\mu) .
\end{gathered}
$$

Поэтому

$$
\sup _{f \in W^{r} L_{q}}\left|R_{n}(f)\right| \leqslant \sup _{f \in W_{\lambda(x)}^{r}{ }^{L} q}\left|R_{n}\left(F_{f}\right)\right| .
$$

Из формул (5) и (6) следует, что

$$
\sup _{f \in W_{\lambda(x)^{L} q}^{r}}\left|R_{n}\left(F_{f}\right)\right|=\sup _{f \in W^{r} L_{q}}\left|R_{n}(f)\right| .
$$

Отсюда получаем, что наилучшая на множестве $W_{\lambda(x)}^{r} L_{q}$ формула (4) 
имеет узлы и веса $x_{k}^{*}, A_{k}^{*} \quad(k=1, \ldots, n)$, а ее оценка совпадает с оценкой (3).

Поскольку $0 \leqslant \lambda(x) \leqslant 1$ для $0 \leqslant x \leqslant 1$, формулы (4) являются формулами вида (2). И обратно, так как каждому значению $t \in[0,1]$ соответствует значение $x \in[0,1]$ такое, что $t=\lambda(x)$, каждая формула (2) является формулой вида (4). Следовательно, множества формул (2) и (4) совпадают, но тогда наилучшая на $W_{\lambda(x)}^{r} L_{q}$ формула (2) совпадает с наилучшей на этом же множестве функций формулой (4). Теорема доказана.

Из этой теоремы, например, следует, что при $\varrho(x)=[x(1-x)]^{-1 / 2}$ наилучшая формула (2) на множестве непрерывных на $[0,1]$ функций $f(x)$ таких, что $f\left(\sin ^{2} \frac{\pi}{2} x\right) \in W^{r} L_{q}$, имеет вид

$$
\int_{0}^{1} \frac{f(x)}{\sqrt{x(1-x)}} d x=\pi \sum_{k=1}^{n} A_{k}^{*} f\left(\sin ^{2} \frac{\pi}{2} x_{k}^{*}\right)+E_{n}(f) .
$$

3 амечание. Вышедоказанная теорема распространяется и на случай, когда рассматриваются квадратурные формулы, использующие производные подынтегральной функции, а также на формулы с частично (или полностью) фиксированными узлами.

Пр и м р. Приведем результаты вычисления интеграла

$\int_{0}^{1} \frac{\operatorname{arctg} x}{\sqrt{x(1-x)}} d x=1,3417069$ по формуле (7) для случая $r=2, q=\infty$;

\begin{tabular}{l|c|c}
\hline$n$ & $\begin{array}{c}\text { Значение } \\
\text { интеграла по (7) }\end{array}$ & Ошибка $\left|E_{n}(f)\right|$ \\
\hline
\end{tabular}

$\begin{array}{rll}4 & 1,3405 & 0,0012 \\ 8 & 1,34156 & 0,00015 \\ 16 & 1,341689 & 0,000017 \\ 32 & 1,3417047 & 0,0000022 \\ 64 & 1,34170665 & 0,0000003\end{array}$

\section{Л И Т Р А Т Р Р А}

1. S a r d, A., Amer. J. Math., LXXI, 80 (1949).

2. Ни кольски й С. М., Квадратурные формулы, М., 1974.

Таллинский политехнический институт
Поступила в редакцию 30/IX 1977 